\title{
Augmented Reality Technology for People Living with Dementia and their Care Partners
}

\author{
Matthew A. Hamilton \\ hamilmat@uregina.ca \\ Department of Computer Science, University of Regina \\ Regina, Saskatchewan, Canada \\ Howard J. Hamilton \\ hamilton@cs.uregina.ca \\ Department of Computer Science, University of Regina \\ Regina, Saskatchewan, Canada
}

\begin{abstract}
We designed and implemented an Augmented Reality system, called the My Daily Routine (MDR) system, to demonstrate technology that may aid people living with dementia and their care partners. Although people with dementia are often dependent on their care partners in their daily lives, their independence may be enhanced using Augmented Reality. The MDR system consists of a website and a HoloLens Augmented Reality (AR) application. The care partner can display and customize reminder content using the website. When wearing a Microsoft HoloLens AR device running MDR, a person with dementia will be able to receive personalized reminders in the form of text, images, videos, displayed three dimensional models, voice messages, or music. Customization controls the choice of reminders and their timing; for example, reminders can be issued when an object is detected, at a certain time, or when a command is voiced. MDR can also display the names of common objects and navigation instructions. Through use of the HoloLens' powerful spatial mapping capabilities and Microsoft's experimental World Locking Tools, the indoor navigation system in MDR is accurate and easy to set up.
\end{abstract}

\section{CCS CONCEPTS}

• Human-centered computing $\rightarrow$ User interface programming; Accessibility systems and tools; $\bullet$ Applied computing $\rightarrow$ Consumer health.

\section{KEYWORDS}

augmented reality, HoloLens, dementia, Alzheimer's, care partner, My Daily Routine, reminder system, configurable content, indoor navigation, object detection

\section{ACM Reference Format:}

Matthew A. Hamilton, Anthony Beug, Howard J. Hamilton, and Wil J. Norton. 2021. Augmented Reality Technology for People Living with Dementia

Permission to make digital or hard copies of all or part of this work for personal or classroom use is granted without fee provided that copies are not made or distributed for profit or commercial advantage and that copies bear this notice and the full citation on the first page. Copyrights for components of this work owned by others than the author(s) must be honored. Abstracting with credit is permitted. To copy otherwise, or republish, to post on servers or to redistribute to lists, requires prior specific permission and/or a fee. Request permissions from permissions@acm.org.

ICVARS '21, March 20-22, 2021, Melbourne, Australia

(C) 2021 Copyright held by the owner/author(s). Publication rights licensed to ACM ACM ISBN 978-1-4503-8932-7/21/03 ..\$15.00

https://doi.org/10.1145/3463914.3463918

\author{
Anthony Beug \\ beug200a@uregina.ca \\ Department of Computer Science, University of Regina \\ Regina, Saskatchewan, Canada \\ Wil J. Norton \\ Department of Computer Science, University of Regina \\ Regina, Saskatchewan, Canada \\ Rina, Saskatchewan, Canada
}

and their Care Partners. In ICVARS '21: International Conference on Virtual and Augmented Reality Simulations, March 20-22, 2021, Melbourne, Australia. ACM, New York, NY, USA, 10 pages. https://doi.org/10.1145/3463914.3463918

\section{INTRODUCTION}

Dementia is a term for describing a "decline in memory, reasoning or other thinking skills," [2] and Alzheimer's is a disease that is the largest contributor to dementia "accounting for $60-80 \%$ of dementia cases" [2]. The number of people who are affected by Alzheimer's and dementia continues to grow, and at the time of writing no cure has been verified. Worldwide, it is estimated that "around 50 million people have dementia, and there are nearly 10 million new cases every year" [31]. In the United States alone, an estimated 5.8 million people who are 65 and older have Alzheimer's with symptoms that affect everyday activities [1]. When symptoms of Alzheimer's start to affect everyday activities, it is then considered mild or early stage dementia, which progresses to moderate or middle stage, and then to severe or late stage $[1,31]$. Here, the term dementia is used to encompass all diseases that cause a decline in memory, including Alzheimer's.

People living with dementia may feel like they are losing their independence [34] because they are too dependent on their care partners. For these reasons, technology that assists people with dementia with their daily routines may be useful. There are times when a care partner may not be available to help or may wish for a rest. In this paper, the term care partner refers to anyone who regularly provides care to the person living with dementia.

We were asked by Eden Care Communities (ECC), the operator of many care homes in Regina, Canada, to develop technology that may eventually aid people with dementia and their care partners. ECC staff have observed that some people with dementia remember to put on their glasses even though they do not remember to activate an emergency button on a device around the neck. Thus, ECC was interested in the Microsoft HoloLens, as a precursor to a glasses-like technology that may be available inexpensively in the future. We worked closely with experts at ECC who deal with people with dementia every day to devise features that would benefit people with dementia and their care partners. The experts stated that the HoloLens would be acceptable for testing on people with dementia, even though it would not be acceptable for practical daily use. The major features that they identified as needed are: maintain 
confidentiality of personal information, answer repetitious questions concerning meal times and similar matters, remind the person of meal times and other events, help the person navigate indoors to the dining room and other rooms, play calming music or videos if the person is stressed, and remind the person of the names of people and objects. They also stated that people with dementia would benefit from hearing familiar voices, such as the voices of their loved ones. They suggested that the care partners would be the best sources of information concerning the severity of the dementia and any other health or personal information. Care partners should be able to configure the MDR system via their cellphones or computers. As well, any information should be maintained even after the HoloLens was turned off.

For a preliminary effort, we decided to exclude answering verbal questions and performing facial recognition because of the time required to develop suitable software and assemble appropriate data sets. We derived the following needs for software from the above requirements: (1) security, (2) flexible reminder system, (3) diverse content displayed in the form of text, images, videos, three dimensional models, voice messages, music, or any other kind of audio, (4) configuration for individuals, (5) object recognition, (6) aid for indoor navigation, and (7) content, configuration, and navigation information maintained across sessions.

To determine whether the HoloLens could form the basis of technology to address the above seven requirements, we developed the My Daily Routine (MDR) system and evaluated its technical performance. The My Daily Routine (MDR) system consists of an Augmented Reality (AR) application (app) and a website with an Application Programming Interface (API). When wearing a Microsoft HoloLens AR device running MDR, a person receives customized reminders that can be triggered in various ways. It can also display the names of common objects and navigation instructions. The indoor navigation system is accurate and easy to set up.

A few studies have reported prototype AR apps to support people with dementia in their daily lives, but apparently none of these previous apps allow the person with dementia to be passive while using the AR device, which reduces the level of cognitive demand. A study on using assistive technology in the homes of people who have dementia found that the technology should play a "...passive role," and a device should intervene "... in an autonomous fashion" [21]. Previous apps also do not allow the care partner to easily customize the content (such as video, images, audio, 3D models, etc.) that will assist the person with dementia in their daily lives [30]. As well, compared to previous apps for similar purposes, MDR provides a more general reminder system and a greater diversity of types of content to display as reminders. Unlike previous apps, it provides indoor navigation, time-based reminders, and video reminders.

The paper covers previous work on AR apps for those with dementia in Section 2. An overview of the MDR system is given in Section 3 along with a comparison to previous apps. In Section 4, the main elements of the MDR software are described. In Section 5, the run-time performance of the MDR app running on the HoloLens is evaluated. In Section 6, conclusions are drawn and suggestions for future work are described.

\section{RELATED WORK}

Virtual Reality (VR) and Augmented Reality can aid people with dementia by offering calming experiences, memory aids, and cognitive stimulation [13]. For example, memory games can be used to try to slow the spread of the disease [3]. Other research has been done on using memory games to assess a person's current cognitive ability [4]. Making use of a combination of smart technologies, including sensor devices and wearable technologies, researchers have focused on providing prompts and reminders according to the context as determined by sensors.

Some apps have also been developed to support care partners. Davis et al. developed Story-Call, a multimedia app for mobile devices that provides support for care partners of people with dementia [5, 6]. Zachos et al. created an app that helps care partners think of creative ways of caring for people with dementia [35].

Research has been performed on AR apps to aid people who have dementia with routine tasks that a care partner might normally do. Existing apps for this purpose are either aimed at accomplishing one particular task or lack flexibility. Here, we describe two ideas that assist a person with dementia with completing a specific task and one idea for completing tasks in general.

The cARe [30] app is intended to assist people who have dementia with tasks around the house, such as cooking. The app was specifically designed to allow care partners to customize the cooking instructions in the form of text and images without any programming knowledge. The user can customize the location where the instructions appear in the world. However, the customization process was not as refined as a care partner may require. The customization process involved using a GUI that exported an XML file with instructions and images, which then had to be copied to the HoloLens. The cARe app has arrows that direct the person to where they should be looking. Drawing constant attention to where the user should be looking or having content follow them is valuable, because people with dementia tend to forget what they were doing and look away. According to the creators of the cARe app, a problem resulted from the presentation of the step-by-step instructions that have to be followed, which required manual input to move to the next or previous step. As found in this study, sometimes the users became confused if they skipped a step or went back to a previous step. It may be that following these steps is too mentally demanding for someone who has dementia. Thus, it may be preferable to have audio or video instructions recorded by their care partners to guide them in using the app.

Another study developed an AR app called the Therapy Lens [25], which displayed holograms to guide people with Alzheimer's through the process of making tea. The researchers observed no statistically noticeable difference between making tea with normal instructions and making tea with HoloLens' AR instructions. Nonetheless, some people struggled with using the device, depending on the current severity of the disease. People who have had the disease longer and were in worse condition, made approximately the same number of errors with or without using the HoloLens app. Therefore, it is preferable if the person with dementia does not have to interact with the device. Additionally, the researchers found that most people could use the voice commands to go to the next step, but some people found the process confusing and wanted 
more information. The big clear text and soothing voice were highly appreciated by the participants because of their vision or hearing problems. However, the researchers also mentioned that allowing more personalization options for the individual might reduce information overload from hologram visuals, text, and audio. Clear text, soothing voice, and detailed personalization are desirable for any apps for people with dementia.

Kanno et al. describe a general reminder system for a mobile device [15]. The proposed app, here referred to as the KLCLL app (based on the initials of the author last names), is intended for patients and their care partners. The location and orientation of the patient will be tracked, and when the patient looks at an object or image, a reminder will be displayed along with GPS location information. If an object is in view of the camera, the app could display a customized textual and audio reminder. If a question is asked, the app could also respond with a textual and audio answer. It is unclear from the paper whether the audio responses were prerecorded or were generated using an automated text-to-speech process. The KLCLL app showed the value of customized reminders and relative positioning of displayed content for people with dementia.

\section{THE MDR SYSTEM}

The MDR system consists of two main parts, an easy-to-use website, which allows comprehensive customization of the content, and a HoloLens AR app. Two types of people will use our system. The first is the care partner, who will use the website and the app to perform initial setup. The second is the person with dementia, who will use the app. The care partner can use the website to display and customize reminder content, including text, audio, images, video, $3 \mathrm{D}$ models, text to speech, navigation, and object detection. The care partner can also configure when the reminder content is displayed, such that it will be displayed in any of three circumstances: (1) at a certain time, (2) in response to a vocal command, or (3) when an object is detected. The care partner will use the HoloLens' hold gesture in the app to open the setup menu, so that the name of the person with dementia can be selected from the end-user drop-down menu. Additionally, in case the person with dementia forgets about a reminder that is being displayed or becomes distracted, the images and video will follow the user's head movements.

Let us compare the features present in MDR and the other apps mentioned in Section 2. As with the cARe [30] app, MDR allows care partners to customize text, audio, and images, and to display them to the person with dementia. We expanded on what the cARe app offers, because with MDR a care partner can record instructions involving multiple steps in video or audio form using a simple website interface. The cARe app provides a line directing attention to an object. In contrast, MDR provides arrows that can guide the person with dementia along a path from any location in the home to any other location, in coordination with any reminder. The clear text, soothing voice, and extensive personalization that are present in the Therapy Lens [25] app inspired us to create similar features in the MDR system. As with the proposed KLCLL app, MDRs reminders are stored on a web server, but our implementation expands on the idea and allows for full customization of when the content is displayed, including by time (which is unique to MDR), or in response to voice commands or object detection. MDR can also recognize 20 types of objects using the Tiny YOLO v2 model (a smaller version of YOLO v2 [23]). In comparison, the KLCLL app appears to be trained to detect a few specific objects.

Table 1 summarizes the features of the MDR system and previous apps. For example, the first line shows that displaying text has been implemented by all four apps and it can be customized by the users of cARe and MDR. The cARe app allows the display of text, audio, and image reminders that can all be customized by the care partner and it responds to vocal commands, which cannot be customized. Therapy Lens allows the display of text, audio, and 3D model reminders, which cannot be customized; it responds to vocal commands, which cannot be customized. It is unclear whether KLCLL has audio or text-to-speech, and if so whether these features can be customized (as indicated by question marks). KLCLL is able to provide GPS location tracking because it runs on a smart phone, whereas the other apps are not able to do so because they run run on the HoloLens, which lacks GPS capabilities. MDR provides all features mentioned except GPS location tracking. All provided features in MDR can be customized except object recognition. MDR is the only app that provides video reminders, time-based reminders, and indoor navigation.

\section{SOFTWARE ELEMENTS}

In this section, we describe the elements of the MDR software that were created to meet the needs identified in Section 1. Where relevant, the tools used to build the website and app are also described. The software was designed with emphasis on ease of customization, so that the care partner can customize the reminders and multimedia content and control when it will be displayed. We also implemented an option to allow the app to trigger reminders based on specific values, which we called situations.

The two main components of the MDR system are the website, which is used by the care partner, and the AR app, which is used by both the person who has dementia and the care partners. In the remainder of this section, the features of these two components that are relevant to helping people with dementia are described.

\subsection{Website}

The website is a critical part of the MDR system. The website allows the user (care partner) to easily create and modify reminders and situations and to upload or record content that will be displayed in the app. Information about a specific end-user (a person with dementia) can be customized, so that they can have the most comfortable and familiar experience possible while using the app.

Website Tools: The website user interface was designed with basic HTML, CSS, and JavaScript using the bootstrap framework and a tool called bootstrap studio. The back end uses MySQL for the database (DB) and a PHP framework called Laravel for accessing the data and providing dynamic elements on the site, such as the loading and saving of content. The user's uploaded files, including video, images, audio, and 3D models, are stored in an Amazon AWS S3 bucket, and the file path to the location in the bucket is stored in the DB. The website is hosted through a shared web hosting service, so that it can be accessed from the HoloLens. 
Table 1: Comparison of Apps for Aiding People with Dementia with Their Daily Lives

\begin{tabular}{lcccc}
\hline \multirow{2}{*}{ Feature } & \multicolumn{4}{c}{ Is the Feature Present? / If So, Can It Be Customized?) } \\
& cARe & Therapy Lens & KLCLL App & My Daily Routine \\
\hline Text & $\mathrm{Y} / \mathrm{Y}$ & $\mathrm{Y} / \mathrm{N}$ & $\mathrm{Y} / \mathrm{N}$ & $\mathrm{Y} / \mathrm{Y}$ \\
Audio & $\mathrm{Y} / \mathrm{Y}$ & $\mathrm{Y} / \mathrm{N}$ & $?$ & $\mathrm{Y} / \mathrm{Y}$ \\
Image & $\mathrm{Y} / \mathrm{Y}$ & $\mathrm{N}$ & $\mathrm{N}$ & $\mathrm{Y} / \mathrm{Y}$ \\
Video & $\mathrm{N}$ & $\mathrm{N}$ & $\mathrm{N}$ & $\mathrm{Y} / \mathrm{Y}$ \\
3D Model & $\mathrm{N}$ & $\mathrm{Y} / \mathrm{N}$ & $\mathrm{N}$ & $\mathrm{Y} / \mathrm{Y}$ \\
Text-to-Speech & $\mathrm{N}$ & $\mathrm{N}$ & $?$ & $\mathrm{Y} / \mathrm{Y}$ \\
Indoor Navigation & $\mathrm{N}$ & $\mathrm{N}$ & $\mathrm{N}$ & $\mathrm{Y} / \mathrm{Y}$ \\
Object Detection & $\mathrm{N}$ & $\mathrm{N}$ & $\mathrm{Y} / \mathrm{N}$ & $\mathrm{Y} / \mathrm{N}$ \\
Time-Based Reminders & $\mathrm{N}$ & $\mathrm{N}$ & $\mathrm{N}$ & $\mathrm{Y} / \mathrm{Y}$ \\
Vocal Commands & $\mathrm{Y} / \mathrm{N}$ & $\mathrm{Y} / \mathrm{N}$ & $\mathrm{Y} / \mathrm{N}$ & $\mathrm{Y} / \mathrm{Y}$ \\
GPS Location Timing & $\mathrm{N}$ & $\mathrm{N}$ & $\mathrm{Y} / \mathrm{Y}$ & $\mathrm{N}$ \\
\hline
\end{tabular}

Security (Website): To provide confidentiality for stored content and configuration information, MDR employs user authentication. The Auth software, which is part of Laravel, handles the user authentication and encryption of passwords. Auth ensures that personal information is protected. Auth is also responsible for connecting an HTTP request to a page and determining if the user is authenticated. Using this authentication, passwords are hashed using the bcrypt algorithm, which adds a salt, making the hash for each password unique with this additional random data [22]. The Auth software increases security because plain-text passwords cannot be seen by those maintaining MDR or by any attacker going through the database.

Configuration for Individuals: The most important purpose of the website is to support configuration of MDR by the care partners. To meet the needs of care companies who may want their employees to manage or create reminder content for multiple people with dementia, MDR can be configured to share content among endusers.

To begin configuring MDR, the user signs in to the website. Signing in reveals sections for Personal Information, Reminders, Situations, and four types of content uploading (see Fig. 1 and Fig. 2). The name of the user is displayed at the top on the far right with a drop-down button for signing out. The drop-down menu to the left of the Add and Remove buttons gives the list of end-users.

Important configuration information is stored in the Personal Information section, which includes details such as if the person is hard of hearing, which affects whether or not audio is played in the app. Additionally, the care partner can add notes and destinations pertaining to a specific end-user in this section.

The Reminders section allows care partners to set reminders to occur at a specific time, via vocal command, upon object detection, or any combination of these three. A reminder can display text, video, images, audio, or 3D models, or it can navigate the user to a destination. The Situations section is for events that are displayed in response to special circumstances, such as detecting that the person with dementia is stressed. The care partner can select or create content that will be familiar to the person with dementia. It has been found that "... persons with dementia preserve long-term memories and positive emotions. Therefore, an effective presentation, such

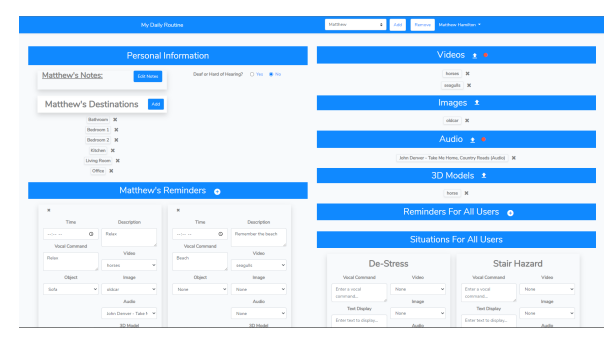

Figure 1: Configuration Web Page

as audiotape contact with a family member, could enhance psychological well-being" [26]. The findings are not limited to just audio, but extend to videos [28] and other content [33] as well.

To simplify the configuration process, the web page is split into a left half, which has local content that pertains to a specific enduser, and a right half, which has global content that applies to all end-users. The left half contains personal information, reminders, and situations that change according to the end-user selected in the drop-down menu. The right half contains reminders and situations for everyone and a section for uploading content that does not change upon selecting a different end-user. The division of the page into two halves provides a logical organization, which should make it easier to use both visually and functionally for care partners to customize content. An example of global content that may be desired is a lunch time reminder that directs every user to where they are supposed to eat. However, if there are different designated eating areas for certain individuals, as is the case with some care home facilities [32], the reminder can be personalized for them.

To improve the user experience with the MDR system, the user is able to record video and audio files directly through the website, name them, and upload them. In this way, a care partner can easily record a video or audio clip of them speaking to the person with dementia, perhaps reminding them to take their medication. Laravel's passport provider adds web API support to Laravel. When correct authentication details are sent from Unity, a Laravel-based route file will call a function in the controller file and return JavaScript Object Notation (JSON) data obtained by a DB query. The route file 


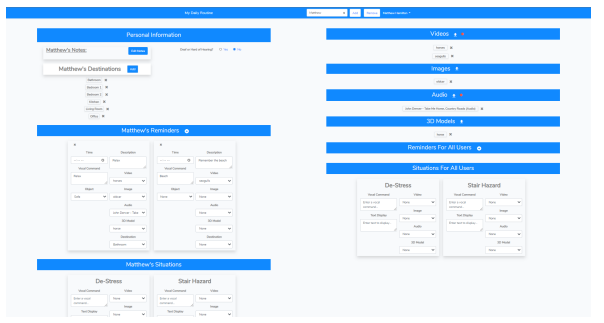

Figure 2: Configuration Web Page (Zoomed Out)

will redirect to the appropriate function based on the specific URL web API request sent by a Unity script.

Configuration for Repetition: For individuals who would benefit from having audio and video repeated or played for longer than usual, an option is provided in the setup menu in the app that allows the user to constrain the content length. There are three options: short, medium, and long. Images and 3D models are displayed for thirty seconds for the short option, one minute for medium, and two minutes for long. As necessary, video and audio recordings are looped. The short option does not loop the content, medium option loops the content two times, and the long option loops the content four times. These options allow an end-user with dementia to have the time they need to process the content.

\subsection{AR Application}

The second major part of the MDR system is the AR app. To begin using the app, the user first logs into the AR app using their website login credentials, and then selects the end-user who will be wearing the HoloLens. Reminders and situations are then displayed on the HoloLens along with the names of detected objects. To describe the $\mathrm{AR}$ app, we first discuss the tools supporting its implementation and then its features.

AR App Tools: The team who works on the Microsoft Mixed Reality Toolkit (MRTK) currently provides more support for the Unity game engine than for the Unreal Engine. The MRTK enables faster development by providing some ready-made scripts, an API to interact with the HoloLens' core functionality, and a built-in tool for deploying to the HoloLens. Therefore the Unity game engine was used to create the AR app. Unity uses Microsoft's C\# programming language and allows developers to create custom logic with scripts for a game or an app. Unity supports assets and plugins, so that external code and functionality can be utilized. For this project, the major third-party assets and plugins utilized were the MRTK[19], the AWS S3 plugin to retrieve user content, TriLib[24] to import 3D models into Unity at run-time, and Microsoft's World Locking Tools (WLTs)[9] for indoor navigation.

Security (App): In order to protect the confidentiality of personal information on the HoloLens, a login menu was created so that the user's credentials can be confirmed before retrieving user content from the website's DB into the app (see Fig. 3). After the user performs the select gesture on an input field, the HoloLens' virtual keyboard will pop up, allowing credentials to be entered.

Diverse Content: We met the need for displaying diverse types of content by using various built-in Unity networking functions and a third-party importer. The networking functions are used

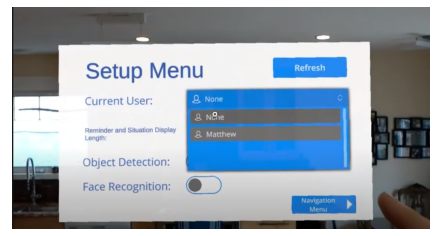

Figure 3: Setup Menu and End-User Drop-down Menu

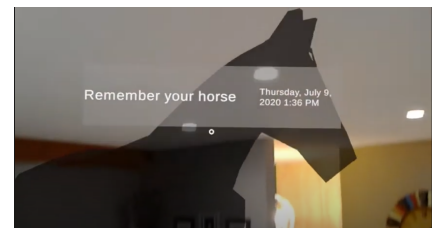

Figure 4: 3D Horse Model Example

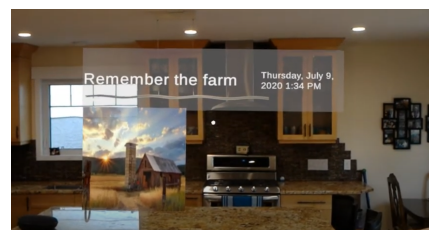

Figure 5: Image Example

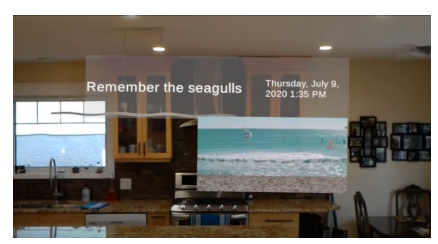

Figure 6: Video Example

to download the files into Unity at run-time using the relevant URLs, whenever reminders or situations are triggered. For example, audio in either OGG or WAV format can be played using Unity's built-in audio player. Unity does not currently provide support for downloading and loading 3D models at run-time, but third party importers are available[7, 24]. Since the TriLib asset supports the Universal Windows Platform (UWP) as well as the importing of more than 40 3D model formats, it was used to import 3D models into the HoloLens at run-time asynchronously. UWP is a standard app platform available on every device that runs Windows 10. Figures 4, 5, and 6 show a model, an image, and a video, respectively. Maintain Content: The need for content to be maintained is satisfied using an external database. To retrieve content via the web API in Unity, each call to the API sends the authentication details and a URL via a web request, with callback functions in-place to handle what happens when the request fails or succeeds. On success, the content, such as reminders or situations, are stored in a JSON object. Then the name of an end-user can be selected from a drop-down list in the setup menu, which is accessed by performing the hold gesture for approximately one second. The hold gesture 


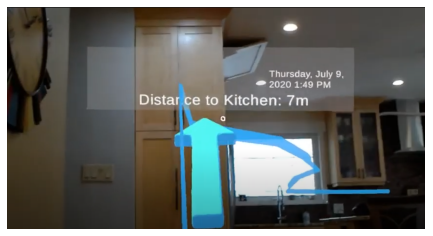

Figure 7: Navigation Example

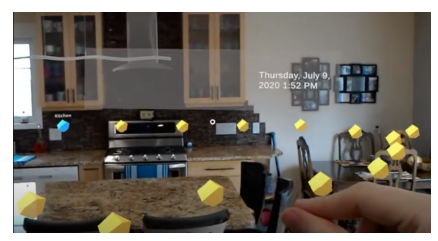

Figure 8: Nodes Example is in-place to prevent the menu from being accidentally opened by the end-user, which might cause confusion. After the name of an end-user has been selected, files from the AWS S3 server are retrieved at run-time using Amazon's new .NET Standard 2.0 SDK, because Amazon no longer supports the Unity specific SDK. To retrieve a URL for each resource from the S3 bucket, authentication information, a bucket id, and a bucket file path are sent. A bucket refers to a file folder or drive stored on the $\mathrm{S} 3$ server. The retrieved URL lasts for a specified period of time before expiring.

Relative Positioning: People who have dementia tend to lose focus, and may look away, so having the content follow their gaze is important [27, 30]. Indeed, having the content follow the user's head is a major advantage of using a head mounted display (HMD) like the HoloLens, as opposed to using a hand-held device. Video and images are displayed using Unity's user interface, and they follow the end-user's head movements using an MRTK solver. In the MRTK, a solver is software that allows one object to follow another in a 3D scene[18]. The solver tracks a part of the end-user, such as the hands or head, and can be set to be in either orbital or radial mode. In orbital mode, the game object, or in this case the video or image, orbits around the user's head with some offset like a planet. In radial mode, a specified portion of the content remains in the user's view, and it is set to stay a minimum and maximum distance away from the head. After 3D models, such as a specific car model, are imported into the HoloLens using TriLib, they are sized appropriately and placed on the floor using a custom script. To determine floor height, the HoloLen's spatial mesh is analyzed to find the lowest bounded plane that has an upward facing normal vector. A plugin from the HoloToolkit (HTK), an older version of the MRTK, is used to find the bounded planes. If no floor planes are detected, we used a default value of -1.1, which was shown by testing to be a reasonable indicator of the floor height.

Indoor Navigation: To meet the need for indoor navigation, a path to a destination can be displayed as part of any reminder (see Fig. 7). Indoor navigation is accomplished by utilizing the HoloLens' spatial mesh mapping, World Locking Tools, $A^{*}$ path finding, and some scripts that handle node or anchor creation. To initialize navigation, the user goes to the navigation menu through the setup menu and then enables the "Navigation Setup Mode". In this mode, as the user walks around, MDR automatically places yellow orb game objects, which are referred to as nodes, in the virtual world (see Fig. 8). The nodes are placed every 0.5 meters $(\mathrm{m})$ as the user walks, but the software allows them to be placed between 0.45 $0.55 \mathrm{~m}$ apart, accounting for the HoloLens' sensor inaccuracy of $\pm 10 \%$. A node is a neighbour of an existing node if it is within $3 \mathrm{~m}$ of it and visible. Visibility is determined by casting a ray between nodes. If the ray does not hit any of the spatial meshes that the
HoloLens automatically creates to model objects in its vicinity, then the objects are considered to be visible to each other. If a ray hits a mesh, an object (such as a wall) is likely blocking the path. To handle cases where the HoloLens has not spatially mapped everything on the first walk through, more ray casts are made at navigation-time between pairs of nearby nodes along the path to a destination. During a navigation-time ray cast, if any neighbour is not visible, then this node is removed from the path and the path is re-calculated.

Maintain Navigation Information: To ensure that a consistent coordinate space is maintained between uses of the HoloLens, World Locking Tools (WLTs) are used [9, 10]. Without WLTs, setup would need to be performed every time the app is launched. For example, if the app were to be launched in the kitchen, then that location would be the zero position for the duration of the app run-time. If the app were to be relaunched in the bedroom, then the bedroom would be the new zero position, and any existing nodes would not be correctly located with respect to their original real-world coordinates. With WLTs, however, world anchors placed in the virtual world prevent this from happening. Each world anchor contains spatial information that locks it to a location in the physical world. When the app is restarted, the nodes in the virtual world are re-aligned to the physical world. In this way, nodes placed during one launch of the app will refer to the same position in the real world when the app is launched again. WLTs also attempt to mitigate the drifting of nodes.

Object Detection: To meet the need to provide the end-user with names of objects, the app can optionally use object detection to supply names for certain objects that the end-user is looking at while wearing the headset. This option can be toggled from the setup menu. Fig. 9 shows an example of a chair and a potted plant being detected. To allow evaluation of the technology, a bounding box is also displayed around the object. The top left of the bounding box shows a label containing the class of object that was detected and the confidence that the bounding box contains that class. When an object is detected, several rays are cast through the bounding box in image space. These rays are cast in an attempt to hit the HoloLens' spatial mesh to determine the depth of the object's bounding box in 3D space. Since the spatial mesh could contain holes, an intersection with the spatial mesh may not be found. To deal with this problem, we cast five rays in a plus pattern and calculate the weighted average of the depths of the points where the rays intersect the spatial mesh. The center ray is given more weight than the other four rays.

Object detection is performed using a tiny version of the Yolo v2 neural network model [23]. The neural network is trained on the Pascal VOC dataset [8], which contains 20 object classes, including bottle, cat, dog, etc. The trained model is stored in Open Neural 


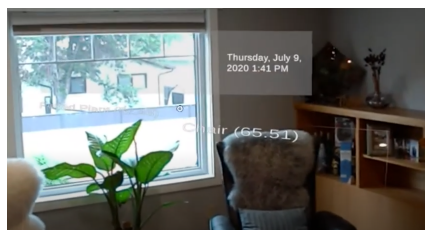

Figure 9: Object Detection Example

Network Exchange (ONNX) format, which is a file format for storing machine learning models. We used the Yolo v2 model available from the ONNX Model Zoo [20]. We used Yolo v2, because Yolo v3 and newer versions are not compatible with the ONNX opset version that the Windows Machine Learning (ML) library uses. The Windows ML library is used to load the ONNX file and evaluate the model on the HoloLens GPU. The evaluation of the model is dispatched on a separate CPU thread instead of the main Unity thread to allow the app to run smoothly while the neural network is being evaluated. Since the evaluation of the neural network can take some time on the HoloLens, the bounding boxes are placed in 3D space with reference to the position of the camera at the time when object detection was started.

\section{PERFORMANCE}

This section discusses the performance of the MDR app. The MDR system has several features running on a HoloLens, so their performance needs to be tested to ensure that they run smoothly together to provide a comfortable experience for the end-user. The performance of the object detection component is of particular importance, because it requires evaluating a neural network model on an image captured at run time, which is computationally demanding. Our solution to the indoor navigation problem should also be evaluated because it is novel. This evaluation is intended to complement previous evaluations of the technical capabilities of the HoloLens $[12,14,17]$, including its navigational capabilities $[11,16,29]$.

We evaluated the performance of the app while performing a variety of tasks. Table 2 shows the eight tasks that were evaluated. As a baseline, we include an Idle task where no reminder content is displayed and no other features such as object detection are enabled. The next five tasks each displayed some text and exactly one of the the other types of content. These tasks are typical of the expected use of the app for displaying reminders and performing navigation. We also evaluated two tasks that we believed were likely to be resource intensive. The Object Detection task only performed object detection. Currently object detection is only enabled when no reminders are playing, to avoid distracting the end-user while the reminder content is shown. The Intensive Reminder task displays as much content as it can. It is intended to reveal the performance limitations of the current implementation.

To evaluate the performance of the app, we first used the Microsoft HoloLens Device Portal's System Performance tool to monitor the app. Fig. 10 shows an example view of the System Performance tool. The tool captured statistics on CPU, memory, I/O, network, power, and GPU usage and frame rate measured in frames

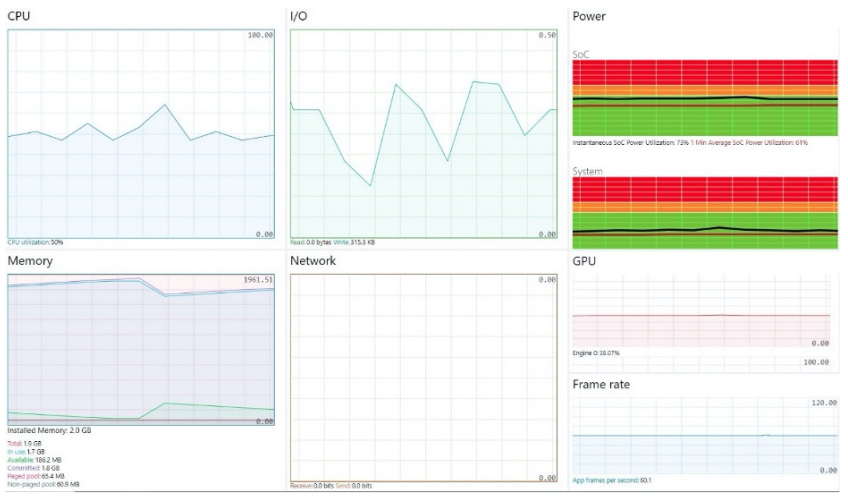

Figure 10: Example Output from the HoloLens System Performance Tool

per second (FPS). Memory was stable at $1.4-1.7$ GB out of 1.9 GB total memory, CPU fluctuated too much to be a useful metric, and $\mathrm{I} / \mathrm{O}$, network, and power usage displayed nothing out of the ordinary. Frame rate is an especially useful metric because it is a good proxy for the smoothness of the experience for the end-user. The maximum FPS that the HoloLens can display is 60 FPS, so that is the target.

Since the System Performance tool has no way to export results, a video of the performance tool was captured and the frames of the video were analyzed to obtain the frame rate reported by the tool at different points in time. This time-consuming evaluation method was performed for the two most resource intensive tasks. An Object Detection task was evaluated while an actual television object was repeatedly detected in the room over a 2-minute period and the average frame rate was calculated for this period. For the Intensive Reminder task, the same reminder was voice activated three times and for each reminder six types of content were simultaneously shown. The average frame rate was calculated during the frames when the reminder was actually being displayed. The results for both tasks are given in Table 3. The observed frame rates of 56.2 and 55.0 FPS are close to the maximum rate of 60 FPS and are adequate for the intended use.

To be thorough, we also measured the frame time and frame rate by capturing the elapsed time using special-purpose code in the app. Time measurements were calculated using a high-resolution performance counter. The Unity game engine has a built-in class called FrameTimingManager that provides accurate CPU and GPU frame timing information, but it is unsupported on Windows, so we were unable to use it. Instead the .NET Stopwatch class was used to query time stamps in a Unity script that runs late each frame, which results in time stamps that are slightly offset from the true end of the frame. The frame times shown here also include any CPU time related to waiting for V-SYNC and time lost to waiting for the GPU to finish executing.

The average frame times and frame rates while performing the tasks given in Table 2 are shown in Table 4. Each average was calculated over 1000 consecutive frames while performing a task. The first six tasks, including indoor navigation, ran at frame rates 
Table 2: Task Characteristics

\begin{tabular}{lccccccc}
\hline Task & $\begin{array}{c}\text { Display } \\
\text { Text }\end{array}$ & $\begin{array}{c}\text { Display } \\
\text { Video }\end{array}$ & $\begin{array}{c}\text { Play } \\
\text { Audio }\end{array}$ & $\begin{array}{c}\text { Display } \\
\text { Image }\end{array}$ & $\begin{array}{c}\text { 3D } \\
\text { Model }\end{array}$ & $\begin{array}{c}\text { Indoor } \\
\text { Navigation }\end{array}$ & $\begin{array}{c}\text { Object } \\
\text { Detection }\end{array}$ \\
\hline Idle & & & & & & & \\
Video + Text & $\mathrm{Y}$ & $\mathrm{Y}$ & & & & & \\
Audio + Text & $\mathrm{Y}$ & & $\mathrm{Y}$ & & & & \\
Image + Text & $\mathrm{Y}$ & & & $\mathrm{Y}$ & & & \\
3 M Model + Text & $\mathrm{Y}$ & & & & $\mathrm{Y}$ & & \\
Indoor Navigation & $\mathrm{Y}$ & & & & & $\mathrm{Y}$ & $\mathrm{Y}$ \\
Object Detection & & & & & & $\mathrm{Y}$ & \\
Intensive Reminder & $\mathrm{Y}$ & $\mathrm{Y}$ & $\mathrm{Y}$ & $\mathrm{Y}$ & $\mathrm{Y}$ & $\mathrm{Y}$ & \\
\hline
\end{tabular}

Table 3: Frame Rate Measurement using the System Performance Tool in the HoloLens Device Portal

\begin{tabular}{lccccc}
\hline & Average FPS & Std Dev & Min FPS & Max FPS & Samples \\
\hline Object Detection & 56.2 & 3.5 & 48.6 & 60 & 112 \\
Intensive Reminder & 55.0 & 6.2 & 44.9 & 60 & 40 \\
\hline
\end{tabular}

close to the maximum of 60 FPS. The Object Detection and Intensive Reminder tasks reduced the frame rate to 48.73 and 44.84 , respectively, which are good rates but not excellent ones. In addition to the minimum and maximum frame time, we also include the $90^{t h}$, $95^{t h}$, and $99^{t h}$ percentiles. If the $99^{t h}$ percentile of a frame time is $t$ milliseconds, then $99 \%$ of all frames were generated within $t$ milliseconds. Overall, MDR is able to display any type of content for a reminder without appreciably slowing down. As well, it can perform object detection or can display multiple types of content for reminders with about a $20 \%$ to $25 \%$ slow down in the frame rate, which may be imperceptible to people.

The elapsed time required to detect an object was evaluated using a similar method. The average time taken to run the object detection on the HoloLens was recorded and the results are shown in Table 5. Averages were taken over 30 consecutive runs of the neural network while looking at a toy car that the neural network can detect. Evaluate includes the time to the run the neural network, which further includes time to set up the input layer of the network by performing bilinear resizing of a captured camera frame using the multithreaded Unity Job System. Process Output includes the time to process the output layer of the neural network, which involves performing non-max suppression to remove overlapping bounding boxes. Total Time is the average time from starting evaluation until the bounding boxes are created in Unity, which includes time to perform ray casting to determine the bounding box locations. Frame Delay is the average number of frames elapsed from start to end of running object detection for a camera frame. These results indicate that it takes an average of 89 frames or about two seconds (1854.60 ms) after an object comes into view before its name is displayed in the HoloLens. In the meantime, the HoloLens continues to show other frames at a rate of 48.73 FPS (See Table 4). This performance should be acceptable for its intended purpose.

\section{CONCLUSIONS}

The MDR system, consisting of a website and a HoloLens AR app, provides a test bed for developing technology for improving the daily lives of those with dementia and their care partners. The system has been designed from the ground up keeping their needs in mind. The MDR system can be extensively customized by the care partners, who may best know what reminders would be most helpful. All content, configuration, and navigation information is maintained between sessions with the HoloLens and kept secure. The performance of the system is stable, achieving an average frame rate of 44.84 to 60 FPS in the tested scenarios. Object detection works reasonably well with an average frame rate of 48.73 FPS. Indoor navigation works well without reducing the frame rate appreciably. No initial map is required.

As noted in the introduction, MDR provides a more general reminder system than previous apps and a greater diversity of types of content to display as reminders than them. Three novel features for this type of app are: indoor navigation, time-based reminders, and video reminders.

In the future, we will develop content suited to those with dementia. We will also conduct a survey to evaluate the user experience of using the MDR system. We will conduct a survey in a care home facility after the COVID-19 pandemic.

The MDR system could be improved in various ways. Question answering and facial recognition could be added to the app. Upon recognizing a face and displaying name, a reminder could be activated; this reminder could vary depending on the person recognized by the software. Our object detection method may be further improved by adding object tracking alongside the detection, so that tracking occurs smoothly rather than taking a second before updated results are processed. A heart rate sensor could be added for situations where the person may be feeling stressed or is working too hard. This could allow reminders to be automatically triggered. Adapting the MDR system to the HoloLens 2 should improve the object detection performance and overall app performance. Future 
Table 4: Frame Time Statistics for the Tasks in Table 2

\begin{tabular}{lcccccccc}
\hline & $\begin{array}{c}\text { Average } \\
\text { Frame } \\
\text { Time }(\mathbf{m s})\end{array}$ & $\begin{array}{c}\text { Std Dev } \\
\text { Frame } \\
\text { Time }(\mathbf{m s})\end{array}$ & $\begin{array}{c}\text { Min } \\
\text { Frame } \\
\text { Time }(\mathbf{m s})\end{array}$ & $\begin{array}{c}\text { Max } \\
\text { Frame } \\
\text { Time (ms) }\end{array}$ & $\begin{array}{c}\mathbf{9 0}^{\text {th }} \\
\text { Perc. }\end{array}$ & $\begin{array}{c}\mathbf{9 5}^{\text {th }} \\
\text { Perc. }\end{array}$ & $\begin{array}{c}\mathbf{9 9}^{\text {th }} \\
\text { Perc. }^{\text {Average }}\end{array}$ & $\begin{array}{c}\text { AvS } \\
\text { FPS }\end{array}$ \\
\hline Idle & 16.68 & 1.55 & 9.52 & 33.03 & 18.11 & 18.67 & 21.75 & 59.96 \\
Video + Text & 16.73 & 3.79 & 9.11 & 45.34 & 21.56 & 24.85 & 29.96 & 59.77 \\
Audio + Text & 16.72 & 2.21 & 10.10 & 37.61 & 18.11 & 18.80 & 27.27 & 59.82 \\
Image + Text & 16.73 & 1.87 & 11.69 & 36.57 & 18.34 & 18.99 & 22.69 & 59.76 \\
3D Model + Text & 16.65 & 1.37 & 11.08 & 39.57 & 17.82 & 18.27 & 20.21 & 60.07 \\
Indoor Navigation & 16.70 & 2.91 & 10.18 & 48.95 & 19.70 & 21.40 & 24.70 & 59.87 \\
Object Detection & 20.52 & 8.46 & 9.57 & 60.17 & 32.22 & 37.98 & 49.72 & 48.73 \\
Intensive Reminder & 22.30 & 4.41 & 11.15 & 71.70 & 25.59 & 27.72 & 40.79 & 44.84 \\
\hline
\end{tabular}

Table 5: Object Detection Performance

\begin{tabular}{lcccc}
\hline & Evaluate $(\mathbf{m s})$ & Process Output $(\mathbf{m s})$ & Total Time $(\mathbf{m s})$ & Frame Delay (frames) \\
\hline Average & 1772.55 & 71.49 & 1854.60 & 89.07 \\
Std Dev & 33.65 & 11.26 & 31.98 & 5.16 \\
\hline
\end{tabular}

versions of the MDR system could be designed for inexpensive, lightweight devices (as they become available) to be accessible and affordable to more people living with dementia.

\section{ACKNOWLEDGMENTS}

We acknowledge ISM Canada and the Natural Sciences and Engineering Research Council of Canada for funding, Eden Care Communities for consultations, and Naran Chirakkal Ruckiya Sinorina and Neha Rajput for editing and formatting assistance.

\section{REFERENCES}

[1] Alzheimer's Association 2020. Alzheimer's Disease Facts and Figures. Alzheimer's Association, Chicago.

[2] Alzheimer's Association 2020. Dementia vs. Alzheimer's Disease: What is the Difference? Alzheimer's Association. https://www.alz.org/alzheimers-dementia/ difference-between-dementia-and-alzheimer-s.

[3] B. Aruanno, F. Garzotto, and M.C. Rodriguez. 2017. HoloLens-based Mixed Reality Experiences for Subjects with Alzheimer's Disease. In 12th Biannual Conference on Italian SIGCHI Chapter (Cagilari). Association for Computing Machinery, New York, 15:1-9.

[4] C. Boletsis and S. McCallum. 2016. Augmented Reality Cubes for Cognitive Gaming: Preliminary Usability and Game Experience Testing. International Journal of Serious Games 3, 1 (2016), 3-18.

[5] B Davis, M Nies, M Shehab, D Shenk, and P Alfonzo. 2014. Developing a pilot e-mobile app for dementia caregiver support: Lessons learned. Online fournal of Nursing Informatics (OFNI) 18, 1 (2014), 13 pages.

[6] BH Davis, M Shehab, D Shenk, and M Nies. 2015. E-mobile pilot for communitybased dementia caregivers identifies desire for security. Gerontechnology 13, 3 (2015), 332-336.

[7] Dummiesman. 2019. Runtime OBJ Importer. https://assetstore.unity.com/ packages/tools/modeling/runtime-obj-importer-49547. Available:

[8] M. Everingham, L.V. Gool, C.K.I. Williams, J. Winn, and A. Zisserman. 2010. The PASCAL Visual Object Classes (VOC) Challenge. International fournal of Computer Vision 88, 2 (2010), 303-338.

[9] M. Finch. 2019. World Locking Tools Concepts. https //github.com/microsoft/MixedReality-WorldLockingTools-Unity/blob/ master/DocGen/Documentation/Concepts.md. [Online]. Available: https://github.com/microsoft/MixedReality-WorldLockingTools-Unity/ blob/master/DocGen/Documentation/Concepts.md.

[10] M. Finch. 2020. Space Pins. https://github.com/microsoft/MixedRealityWorldLockingTools-Unity/blob/master/DocGen/Documentation/Concepts/ Advanced/SpacePins.md. [Online]. Available: https://github.com/
microsoft/MixedReality-WorldLockingTools-Unity/blob/master/DocGen/ Documentation/Concepts/Advanced/SpacePins.md.

[11] R.L.M. Guarese and A. Maciel. 2019. Development and Usability Analysis of a Mixed Reality GPS Navigation Application for the Microsoft HoloLens. In Advances in Computer Graphics (CGI 2019) (Calgary). Springer, Cham, 431-437. https://doi.org/10.1007/978-3-030-22514-8 41

[12] A. Hamacher, J. Hafeez, R. Csizmazia, and T. Whangbo. 2019. Augmented Reality User Interface Evaluation - Performance Measurement of Hololens, Moverio and Mouse Input. International Association of Online Engineering. https: //www.learntechlib.org/p/208272/ [Online]. Available: https://www.learntechlib. org/p/208272/.

[13] Jason Hayhurst. 2018. How Augmented Reality and Virtual Reality is Being Used to Support People Living with Dementia: Design Challenges and Future Directions. In Augmented Reality and Virtual Reality. Springer, Cham, 295-305. https://doi.org/10.1007/978-3-319-64027-3_20

[14] Patrick Hübner, Kate Clintworth, Qingyi Liu, and Martin Weinmann. 2020. Evaluation of HoloLens Tracking and Depth Sensing for Indoor Mapping Applications. Sensors 20, 4 (2020), 1021.

[15] K.M. Kanno, E.A. Lamounier, A. Cardoso, E.J. Lopes, and G.F.M. Lima. 2018. Augmented Reality System for Aiding Mild Alzheimer Patients and Caregivers. In 2018 IEEE Conference on Virtual Reality and 3D User Interfaces (VR 2017) (Reutlingen). IEEE, New York, 593-594.

[16] Sebastian Kasprzak, Andreas Komninos, and Peter Barrie. 2013. Feature-Based Indoor Navigation Using Augmented Reality. In Proceedings of the 2013 9th International Conference on Intelligent Environments (IE '13). IEEE Computer Society, USA, 100-107. https://doi.org/10.1109/IE.2013.51

[17] Y. Liu, H. Dong, L. Zhang, and A.E. Saddik. 2018. Technical Evaluation of HoloLens for Multimedia: A First Look. IEEE MultiMedia 25, 4 (2018), 8-18. https://doi. org/10.1109/MMUL.2018.2873473

[18] Microsoft 2020. Creating Dynamic Content using Solvers. Microsoft. https://docs.microsoft.com/en-us/windows/mixed-reality/develop/unity/ tutorials/mr-learning-base- 05

[19] Microsoft 2021. Mixed Reality Documentation. Microsoft. https://docs.microsoft. com/en-us/windows/mixed-reality/

[20] ONNX Community. 2020. ONNX Model Zoo. [Online]. Available: github.com/ onnx/models.

[21] R. Orpwood, C. Gibbs, T. Adlam, R. Faulkner, and D. Meegahawatte. 2005. The design of smart homes for people with dementia-user-interface aspects. Universal Access in the Information Society 4, 2 (2005), 156-164.

[22] N. Provos and D. Mazieres. 1999. A Future-Adaptable Password Scheme. In USENIX Annual Technical Conference. Usenix, Monterey, 32 pages.

[23] J. Redmon and A. Farhadi. 2017. YOLO9000: Better, Faster, Stronger. In IEEE Conference on Computer Vision and Pattern Recognition (CVPR 2017). IEEE, Honolulu, 6517-6525.

[24] R. Reis. 2020. TriLib - Model loader package. https://assetstore.unity.com/ packages/tools/modeling/trilib-model-loader-package-91777. Available:. 
[25] Nina Rohrbach, Philipp Gulde, Alan Robert Armstrong, Linda Hartig, Anas Abdelrazeq, Stefan Schröder, Johanne Neuse, Timo Grimmer, Janine Diehl-Schmid, and Joachim Hermsdörfer. 2019. An augmented reality approach for ADL support in Alzheimer's disease: A crossover trial. NeuroEngineering and Rehabilitation 16 , 1 (2019), 1-11.

[26] G. Son, B. Therrien, and A. Whall. 2002. Implicit Memory and Familiarity Among Elders with Dementia. Journal of Nursing Scholarship 34, 3 (2002), 263-267.

[27] C.L. Stopford, J.C. Thompson, D. Neary, A.M. Richardson, and J.S. Snowden. 2012 Working memory, attention, and executive function in Alzheimer's disease and frontotemporal dementia. Cortex 48, 4 (2012), 429-446.

[28] S. Sävenstedt, C. Brulin, and P.-O. Sandman. 2003. Family members' narrated experiences of communicating via video-phone with patients with dementia staying at a nursing home. Fournal of Telemedicine and Telecare 9, 4 (2003), 216-220.

[29] Prashant Verma, Kushal Agrawal, and V. Sarasvathi. 2020. Indoor Navigation Using Augmented Reality. In Proceedings of the 2020 4th International Conference on Virtual and Augmented Reality Simulations (Sydney, NSW, Australia) (ICVARS 2020). Association for Computing Machinery, New York, NY, USA, 58-63. https: //doi.org/10.1145/3385378.3385387

[30] D. Wolf, D. Besserer, K. Sejunaite, M.W. Riepe, and E. Rukzio. 2018. cARe: An Augmented Reality Support System for Dementia Patients. In 31st User Interface
Software and Technology Symposium. Association for Computing Machinery, Berlin, 42-44.

[31] World Health Organization 2019. Dementia. World Health Organization. https: //www.who.int/news-room/fact-sheets/detail/dementia.

[32] R. Wrublowsky. 2018. Design Guide for Long Term Care Homes. https://www.fgiguidelines.org/wp-content/uploads/2018/03/MMP DesignGuideLongTermCareHomes_2018.01.pdf. [Online]. Available: https://www.fgiguidelines.org/wp-content/uploads/2018/03/MMP DesignGuideLongTermCareHomes_2018.01.pdf.

[33] C. Yamagata, M. Kowtko, J.F. Coppola, and S. Joyce. 2013. Mobile app development and usability research to help dementia and Alzheimer patients. In IEEE Long Island Systems, Applications and Technology Conference. IEEE, Farmingdale, 1-6.

[34] L. Yates, E. Csipke, E. Moniz-Cook, P. Leung, H. Walton, G. Charlesworth, A. Spector, E. Hogervorst, G. Mountain, and M. Orrell. 2019. The development of the Promoting Independence in Dementia (PRIDE) intervention to enhance independence in dementia. Clinical Interventions in Aging 14 (2019), 1615-1630.

[35] Kos Zachos, Neil Maiden, Kristine Pitts, Sara Jones, Ian Turner, Malcolm Rose, Kevin Pudney, and Julie MacManus. 2013. A software app to support creativity in dementia care. In Proceedings of the 9th ACM Conference on Creativity \& Cognition. Association for Computing Machinery, New York, 124-133. 